\title{
DETERMINAÇÃO DE CARBAMATO DE ETILA EM AGUARDENTES DE CANA POR CG-EM
}

\author{
Renata Adriana Labanca e Maria Beatriz Abreu Glória* \\ Departamento de Alimentos, Faculdade de Farmácia, Universidade Federal de Minas Gerais, Av. Antônio Carlos, 6627, 31270-901 \\ Belo Horizonte - MG, Brasil \\ Robson José de Cássia Franco Afonso \\ Departamento de Química, Universidade Federal de Ouro Preto, R. Diogo de Vasconcelos, 122, 35400-000 Ouro Preto - MG, \\ Brasil
}

Recebido em 28/8/07; aceito em 11/4/08; publicado na web em 1/10/08

\begin{abstract}
DETERMINATION OF ETHYL CARBAMATE IN SUGAR CANE SPIRITS BY GC-MS. A simple, specific and sensitive GCMS procedure with ion $\mathrm{m} / \mathrm{z} 62$ was validated for the determination of ethyl carbamate (EC) in spirits. It exhibited linearity over the concentration of 30 to $600 \mu \mathrm{g} / \mathrm{L}$ with $30 \mu \mathrm{g} / \mathrm{L}$ limit of quantification. EC was detected in 70 of the 71 samples analyzed with levels from 33 to $2609 \mu \mathrm{g} / \mathrm{L}$ (mean level $=893 \mu \mathrm{g} / \mathrm{L}$ ). $35 \%$ of the samples contained 500 to $1000 \mu \mathrm{g} / \mathrm{L}$ and $23 \%$ contained 150 to 500 and 1000 to $1500 \mu \mathrm{g} / \mathrm{L}$. No significant correlation was found between EC and the levels of copper, $\mathrm{pH}$ and alcohol content of the samples.
\end{abstract}

Keywords: sugar cane spirit; ethyl carbamate; GC-MS.

\section{INTRODUÇÃO}

O Brasil destaca-se na produção de aguardente de cana-de-açúcar, com uma produção anual de 1,6 bilhões de L. O grande desafio dos produtores tem sido aprimorar a qualidade, agregar valor ao produto, obter reconhecimento internacional e aumentar as exportações. ${ }^{1,2}$ Estudos recentes têm indicado a melhoria da qualidade em relação aos teores de cobre. ${ }^{3-5}$ Entretanto, o carbamato de etila vem sendo apontado como um preocupante contaminante em aguardente de cana, podendo causar sérios problemas à saúde do consumidor e impor barreiras à exportação.

$\mathrm{O}$ carbamato de etila $\left(\mathrm{H}_{2} \mathrm{NCOOC}_{2} \mathrm{H}_{5}\right)$, também denominado uretana, é o éster etílico do ácido carbâmico. Estudos têm comprovado que este composto é carcinogênico em diversas espécies animais, incluindo ratos, camundongos, hamsters e macacos. Em testes de toxicidade com ratos, o carbamato de etila causou um aumento dose dependente de carcinomas no fígado, pulmões, pele e estômago e de tumores do ovário e da glândula mamária. ${ }^{6}$ Desta forma, o carbamato de etila foi classificado como um possível agente cancerígeno (Classe 2 B) pela Agência Internacional de Pesquisa sobre Câncer, ${ }^{7}$ devendo sua presença em alimentos ser a menor possível. Além de carcinogênico, o carbamato de etila e seu metabólito carbamato de vinila causam mutação em diversos órgãos, incluindo pulmões, fígado e glândulas mamárias. ${ }^{8,9}$

Em 1985, o governo canadense fixou limites para o carbamato de etila em diversos tipos de bebidas fermentadas de 30, 150 e 400 $\mu \mathrm{g} / \mathrm{L}$ em vinho de mesa, bebidas destiladas e aguardente de frutas, respectivamente..$^{10}$ Nos Estados Unidos, o Food and Drug Administration elaborou um plano de redução dos níveis, sendo os limites para uísques de até $125 \mu \mathrm{g} / \mathrm{L}$ e para vinhos de mesa de $15 \mu \mathrm{g} / \mathrm{L} .{ }^{11}$ No Brasil, a presença de carbamato de etila em aguardentes foi regulamentada pela Instrução Normativa $N^{\circ} 13$ de 20 de junho de 2005, que determina um teor máximo de $150 \mu \mathrm{g} / \mathrm{L}$ para aguardentes de cana, estabelecendo um prazo de 5 anos para adequação e controle do contaminante. ${ }^{12}$

\footnotetext{
*e-mail: beatriz@ farmacia.ufmg.br
}

O mecanismo de formação de carbamato de etila em bebidas alcoólicas ainda não é bem compreendido. Em vinhos, os precursores são uréia, carbamilfosfato, arginina e citrulina, formados durante as fermentações alcoólica e malolática. Estes podem reagir com o etanol em pH ácido, formando o carbamato de etila, sendo a reação favorecida pelo aumento da concentração dos reagentes, do tempo e da temperatura. ${ }^{10,13-16}$

Em destilados, a formação de carbamato de etila pode ocorrer antes, durante e depois do processo de destilação, incluindo o armazenamento da bebida. A formação antes da destilação ocorre pelas mesmas vias em vinhos. Entretanto, a contribuição destes para o produto final não deveria ser considerável, uma vez que o ponto de ebulição do carbamato de etila é $185^{\circ} \mathrm{C}$ e o processo de destilação removeria quase por completo este composto. ${ }^{17} \mathrm{O}$ mais importante precursor para a formação do carbamato de etila em destilados parece ser o íon cianeto. Este pode ser formado pela decomposição térmica ou enzimática de glicosídeos cianogênicos. O cianeto é oxidado a cianato em reação catalisada pelo $\mathrm{Cu}^{\mathrm{II}}$ ou por radicais livres provenientes da oxidação de compostos insaturados pela luz UV. O cianato reage com etanol e forma carbamato de etila. A configuração do sistema, o processo de destilação e as condições de armazenamento afetam de forma significativa a formação de carbamato de etila em aguardentes, sendo os teores maiores em temperaturas mais elevadas e taxas de refluxo inadequadas. ${ }^{14,17-19}$

Devido ao risco para a saúde dos consumidores, há necessidade de monitoramento constante dos teores de carbamato de etila de forma segura e confiável..$^{20}$ Inúmeras técnicas têm sido descritas na literatura para a análise de carbamato de etila em bebidas alcoólicas. A mais utilizada é a cromatografia em fase gasosa acoplada a diferentes detectores, dentre eles, espectrômetro de massas - CG-EM, ${ }^{14,21-23}$ detecção seletiva para nitrogênio/fósforo, ${ }^{24,25}$ detector eletrolítico de condutividade de Hall, ${ }^{26}$ detector de infravermelho por transformada de Fourier, ${ }^{27}$ e detecção por luminescência. ${ }^{28}$ O CG-EM tem sido preferido por ser mais seletivo, rápido, e não requerer purificação e extração da amostra.

Este trabalho teve como objetivo avaliar a qualidade de aguardentes de cana produzidas ou comercializadas no estado de Minas 
Gerais com relação aos teores de carbamato de etila. Os objetivos específicos foram validar metodologia por GC-EM para a quantificação de carbamato de etila em aguardente de cana; determinar os teores de carbamato de etila em amostras de aguardente de cana, e correlacionar os teores de carbamato de etila com alguns parâmetros de qualidade, dentre eles, teores de cobre, $\mathrm{pH}$ e grau alcoólico.

\section{PARTE EXPERIMENTAL}

\section{Amostras}

Amostras de aguardente de cana produzidas e/ou engarrafadas no estado de Minas Gerais (total de 71) foram coletadas aleatoriamente no mercado consumidor de Belo Horizonte, MG, no período de maio de 2003 a março de 2004.

\section{Reagentes e padrões}

A maioria dos reagentes era grau analítico e os para a cromatografia eram grau cromatográfico. A água foi destilada e purificada em Milli-Q (Millipore, Billerica, MA, EUA). Carbamato de etila e carbamato de metila, ambos com $99,0 \%$ de pureza foram adquiridos da Sigma (St Louis, MO, EUA).

\section{Otimização de metodologia para a determinação de carbamato de etila em aguardente}

Um método utilizando cromatografia gasosa acoplada à espectrometria de massas foi otimizado e validado para a determinação de carbamato de etila em aguardente de cana. Foi utilizado um cromatógrafo gasoso com detector de espectrometria de massas por ion trap (GC-QMS, Finnigan, Thermo-Fisher Scientific, Waltham, MA, EUA).

Vários testes foram realizados para determinar as condições ótimas de trabalho, dentre elas, o tipo de coluna capilar (Supelcowax - $60 \mathrm{~m}, 0,25 \mathrm{~mm}$ e $0,33 \mu \mathrm{m} ;{ }^{29} \mathrm{CP}$ Wax $52 \mathrm{CB}$ Chrompack - 25 $\mathrm{m}, 0,31 \mathrm{~mm}$ e $0,21 \mu \mathrm{m} ;{ }^{26}$ e Carbowax $20 \mathrm{M}-25 \mathrm{~m}, 0,22 \mathrm{~mm}$ e 0,25 $\mu \mathrm{m}) ;{ }^{28} \mathrm{o}$ íon de massa para monitoramento $(\mathrm{m} / \mathrm{z}, 44,62,74,75,89)$; a temperatura do injetor $\left(220\right.$ a $\left.260{ }^{\circ} \mathrm{C}\right)$; a temperatura da interface $\left(220\right.$ a $230{ }^{\circ} \mathrm{C}$ ) com variação de 5 em $5^{\circ} \mathrm{C}$ e, o volume de amostra injetado $(0,5$ a $2,0 \mu \mathrm{L}) \cdot{ }^{17,23,30,31}$ Foram padronizados o modo splitless e o fluxo do gás de arraste, hélio a $1,5 \mathrm{~mL} / \mathrm{min}$.

De forma a definir qual seria o melhor parâmetro de quantificação (área ou altura do pico), a linearidade da curva analítica, o perfil cromatográfico e a recuperação de amostras adicionadas de carbamato de etila foram verificados.

\section{Validação de metodologia}

Para validação da metodologia foram investigados os parâmetros especificidade, linearidade, limite de detecção do equipamento, limites de detecção e de quantificação do método, exatidão e precisão.

A capacidade do método em determinar especifica e seletivamente o analito de interesse na presença de outros componentes da matriz foi investigada. Isto foi feito com amostras adicionadas do padrão, tendo sido observadas a resolução do pico de carbamato de etila e a presença de picos interferentes.

Foram realizados ensaios com soluções padrão para determinação da linearidade sem interferência da matriz. ${ }^{20}$ As soluções padrão de carbamato de etila foram preparadas em $40 \%$ de etanol nas concentrações de $0,30,50,100,300,400$ e $600 \mu \mathrm{g} / \mathrm{L}$. Para a construção da curva analítica, as soluções foram adicionadas de $500 \mu \mathrm{g} / \mathrm{L}$ do padrão interno, sendo que cada uma foi injetada três vezes de forma aleatória.
Por meio de regressão linear, foram determinados a equação da curva e o coeficiente de correlação.

Para determinar o limite de detecção do equipamento, foram injetadas soluções padrão em concentrações cada vez menores a fim de se estabelecer a menor concentração capaz de emitir um sinal no mínimo três vezes o valor do maior sinal de ruído. ${ }^{20}$ Para determinar o limite de detecção do método foram injetadas amostras da matriz de aguardente de cana adicionadas de padrão em concentrações cada vez menores para determinar a menor concentração capaz de emitir um sinal pelo menos três vezes maior que o sinal obtido para o ruído. Os percentuais de recuperação e os coeficientes de variação foram comparados aos valores recomendados pela Eurachem e pelo Codex..$^{20,32}$

O limite de quantificação do método foi determinado injetandose amostras da matriz adicionadas de padrão em concentrações cada vez menores e estes foram comparados aos limites recomendados pela Eurachem. ${ }^{20}$

A exatidão e precisão do método foram determinadas por meio da recuperação média e do coeficiente de variação, respectivamente, das amostras (matriz) adicionadas de concentrações conhecidas do padrão.

\section{Determinação de carbamato de etila em amostras de aguardentes de cana}

A determinação do carbamato de etila nas aguardentes foi feita em um CG-EM por quadrupolo (modelo CG-17A e 5050-A, Shimadzu, Kyoto, Japão), sendo utilizadas as condições cromatográficas definidas na validação do método.

As amostras de aguardente de cana $(n=71)$ foram analisadas diretamente. $\mathrm{O}$ carbamato de etila foi identificado por comparação do tempo de retenção entre o padrão e as amostras. A confirmação foi feita pela adição de padrão à amostra e pela comparação dos espectros de massas obtidos com o espectro do padrão nas mesmas condições. A quantificação do carbamato de etila foi feita por interpolação em curva analítica e corrigida pelo percentual de recuperação do padrão interno, carbamato de metila. Todas as análises foram feitas em duplicata. As amostras foram analisadas diretamente. As amostras cujas leituras extrapolaram a curva analítica foram diluídas com etanol $40 \%$ e submetidas novamente à análise.

Os resultados foram analisados individualmente e agrupados em quatro regiões - Metropolitana, Noroeste, Sudoeste e Leste - sendo que as duas últimas englobavam algumas macrorregiões propostas pelo IBGE. ${ }^{33}$ A região Sudoeste incluiu as regiões Sul de Minas, Alto São Francisco e Triângulo Mineiro e a Leste incluiu as regiões Vale do Jequitinhonha, Zona da Mata e Rio Doce. Os dados foram submetidos à análise estatística descritiva e análise de variância, sendo as médias obtidas para cada região comparadas pelo teste de Duncan a 5\% de probabilidade.

\section{Correlação entre os teores de carbamato de etila com outros parâmetros de qualidade da aguardente de cana}

Os valores de $\mathrm{pH}$, grau alcoólico e os teores de cobre nas mesmas amostras foram determinados segundo metodologias da AOAC, ${ }^{34} \mathrm{e}$ descritos por Labanca et al.. ${ }^{5}$ Estes resultados e os teores de carbamato de etila foram submetidos à correlação de Pearson em nível de $5 \%$ de significância.

\section{RESULTADOS E DISCUSSÃO}

Otimização da metodologia para a quantificação de carbamato de etila em aguardente de cana

As melhores condições para a determinação de carbamato de etila por 
CG-EM incluíram temperatura do injetor de $250^{\circ} \mathrm{C}$ e da interface de 230 ${ }^{\circ} \mathrm{C}$, programação da temperatura do forno de $90^{\circ} \mathrm{C}(2 \mathrm{~min})$, elevada a 10 ${ }^{\circ} \mathrm{C} / \mathrm{min}$ até $150{ }^{\circ} \mathrm{C}(0 \mathrm{~min})$, e elevada a $40{ }^{\circ} \mathrm{C} / \mathrm{min}$ até $230^{\circ} \mathrm{C}(10 \mathrm{~min})$. Dentre as três colunas testadas, a capilar de fase polar polietilenoglicol Carbowax 20M ( $25 \mathrm{~m}, 0,22 \mathrm{~mm} \mathrm{e} 0,25 \mu \mathrm{m})$ foi a selecionada por apresentar melhor resolução para o carbamato de etila. O volume ideal de injeção foi de 1,0 $\mu \mathrm{L}$ no modo splitless com o tempo de abertura da válvula de purga de $60 \mathrm{~s}$ e o fluxo do gás de arraste, hélio, a 1,5 mL/min.

Para monitoramento foi selecionado o íon de massa $\mathrm{m} / \mathrm{z}, 62$, por ser o mais específico, apresentar intensidade relativa razoável e menos interferentes na determinação de carbamato de etila em aguardente (Figura 1)

(a)

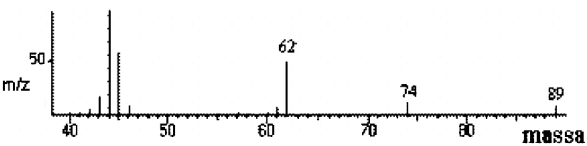

(b)

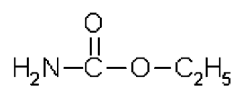

Figura 1. Espectro de massas (a) e fórmula molecular (b) do carbamato de etila

Conforme sugerido por Nagato et al., ${ }^{14} \mathrm{o}$ carbamato de metila foi usado como padrão interno. Além de possuir estrutura química semelhante à do analito de interesse, e eluir adequadamente da coluna separado de todos os outros compostos da amostra e próximo ao carbamato de etila, o carbamato de metila apresentou estabilidade nas condições de análise, forneceu maior intensidade de resposta e $\mathrm{m} / z 75$ como o principal íon ionizável.

Para a determinação do melhor parâmetro para quantificação altura ou área do pico - foram comparados a linearidade de curvas de calibração, os percentuais de recuperação e o coeficiente de variação das respostas de amostras adicionadas de padrão. Maiores coeficientes de correlação foram observados para a altura quando comparados à área (Tabela 1). Entretanto, os picos de carbamato de etila e de carbamato de metila não eram totalmente simétricos. Desta forma, os percentuais de recuperação em amostras adicionadas de diferentes concentrações de carbamato de etila foram determinados. Um maior percentual de recuperação e um menor coeficiente de variação foram obtidos com os cálculos efetuados utilizando-se a área, sendo esta selecionada para a quantificação de carbamato de etila (Tabela 2).

\section{Validação da metodologia para a quantificação de carbamato de etila em aguardente de cana por CG-EM}

No processo de validação da metodologia, observou-se que o método demonstrou ser seletivo, pois foi capaz de determinar exata e especificamente o carbamato de etila na presença de outros componentes presentes na matriz aguardente de cana (Figura 2).

A linearidade da resposta do equipamento foi confirmada na faixa de 30 a $600 \mu \mathrm{g} / \mathrm{L}$ de carbamato de etila em $40 \%$ etanol, baseada na área do pico em 3 dias consecutivos, com coeficientes de correlação superiores a 0,9694 . Não foi constatada a presença de pontos outliers pelo teste de Jacknife padronizado.

Os limites de detecção do equipamento e do método foram de $10 \mathrm{e}$ $30 \mu \mathrm{g} / \mathrm{L}$, respectivamente, por apresentarem relação sinal/ruído maior que $3,0,{ }^{20}$ conforme indicado na Tabela 3 . O limite de quantificação do método de $30 \mu \mathrm{g} / \mathrm{L}$ foi confirmado pelos percentuais de recuperação e coeficientes de variação de 108 e $10 \%$ (Tabela 2), respectivamente, valores aceitáveis pela Eurachem. ${ }^{20}$
Tabela 1. Equações das retas das curvas analíticas e respectivos coeficientes de determinação para carbamato de etila em diferentes dias de análise utilizando-se a área e a altura dos picos obtidos por CG-EM

\begin{tabular}{|c|c|c|}
\hline \multirow{2}{*}{ Dias } & \multicolumn{2}{|c|}{ Equação da reta (coeficiente de determinação, $\mathrm{r}^{2}$ ) / pico } \\
\hline & área & altura \\
\hline 1 & $\begin{aligned} \mathrm{y}= & 0,0005 \mathrm{x}-0,0058 \\
& \left(\mathrm{r}^{2}=0,9884\right)\end{aligned}$ & $\left(r^{2}=(\right.$ \\
\hline 2 & $\begin{aligned} y= & 0,0005 x-0,0054 \\
& \left(r^{2}=0,9899\right)\end{aligned}$ & $\begin{array}{r}y=0,00( \\
\left(r^{2}=\right.\end{array}$ \\
\hline 3 & $\begin{array}{c}\mathrm{y}=0,0005 \mathrm{x}+0,0202 \\
\left(\mathrm{r}^{2}=0,9694\right)\end{array}$ & $\begin{array}{r}y=0,000 \\
\left(r^{2}=\right.\end{array}$ \\
\hline \multicolumn{3}{|c|}{$\begin{array}{l}\mathrm{y}=\text { área ou altura do pico; } \mathrm{x}=\text { concentração do analito em } \mu \mathrm{g} / \mathrm{mL} \\
\text { GC-EM em coluna Carbowax } 20 \mathrm{M}(25 \mathrm{~m} \text { x } 0,22 \mathrm{~mm} \times 0,25 \mu \mathrm{m}) \\
\text { temperaturas do injetor e da interface de } 250 \text { e } 230{ }^{\circ} \mathrm{C} \text {, respectiva- } \\
\text { mente; temperatura do forno de } 90{ }^{\circ} \mathrm{C} / 2 \mathrm{~min} \text {, elevada a } 10{ }^{\circ} \mathrm{C} / \mathrm{min} \\
\text { até } 150{ }^{\circ} \mathrm{C}(0 \mathrm{~min}) \text { e elevada a } 40{ }^{\circ} \mathrm{C} / \mathrm{min} \text { até } 230{ }^{\circ} \mathrm{C}(10 \mathrm{~min} \text {. }) \text { gás } \\
\text { de arraste }-\mathrm{He} \text { a } 1,5 \mathrm{~mL} / \mathrm{min} \text {; volume de injeção } 1,0 \mu \mathrm{L} \text {; íons moni- } \\
\text { torados } \mathrm{m} / \mathrm{z} \text {. } 62 \text { e } 75 \text {. }\end{array}$} \\
\hline
\end{tabular}

Tabela 2. Percentuais de recuperação e coeficientes de variação de carbamato de etila em amostras de aguardente de cana adicionadas de diferentes concentrações de padrão e quantificados utilizando-se área e altura dos picos obtidos por CG-EM

\begin{tabular}{|c|c|c|c|c|}
\hline \multirow{3}{*}{$\begin{array}{l}\text { Padrão } \\
\text { adicionado } \\
(\mathrm{mg})\end{array}$} & \multicolumn{4}{|c|}{ Percentual (\%) } \\
\hline & \multicolumn{2}{|c|}{ Recuperação } & \multicolumn{2}{|c|}{ Coeficiente de variação } \\
\hline & Área & Altura & Área & Altura \\
\hline 30 & 108 & 109 & 10 & 7,0 \\
\hline 100 & 110 & 114 & 6,0 & 8,4 \\
\hline 150 & 107 & 76,7 & 3,8 & 3,9 \\
\hline 400 & 107 & 78,8 & 0,2 & 5,6 \\
\hline 600 & 91,9 & 85,4 & 2,5 & 8,1 \\
\hline
\end{tabular}

GC-EM em coluna Carbowax 20M (25 m x 0,22 mm x 0,25 $\mu \mathrm{m})$; temperaturas do injetor e da interface de 250 e $230{ }^{\circ} \mathrm{C}$, respectivamente; temperatura do forno de $90{ }^{\circ} \mathrm{C} / 2 \mathrm{~min}$, elevada a $10{ }^{\circ} \mathrm{C} / \mathrm{min}$ até $150{ }^{\circ} \mathrm{C}(0 \mathrm{~min})$ e elevada a $40{ }^{\circ} \mathrm{C} / \mathrm{min}$ até $230{ }^{\circ} \mathrm{C}(10 \mathrm{~min}$.); gás de arraste $-\mathrm{He}$ a $1,5 \mathrm{~mL} / \mathrm{min}$; volume de injeção $1,0 \mu \mathrm{L}$; íons monitorados $m / z$, 62 e 75 .

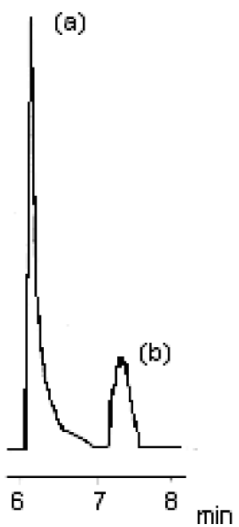

Figura 2. Parte do cromatograma com os picos de carbamato de metila (a) e carbamato de etila (b) em amostra de aguardente de cana. GC-EM em coluna Carbowax 20 M (25 m x 0,22 mm x 0,25 $\mu \mathrm{m})$; temperaturas do injetor e da interface de 250 e $230^{\circ} \mathrm{C}$, respectivamente; temperatura do forno de $90{ }^{\circ} \mathrm{C} / 2$ min, elevada a $10^{\circ} \mathrm{C} / \mathrm{min}$ até $150^{\circ} \mathrm{C}(0 \mathrm{~min})$ e elevada a $40{ }^{\circ} \mathrm{C} /$ min até $230^{\circ} \mathrm{C}$ (10 min); He a 1,5 $\mathrm{mL} / \mathrm{min}$; volume de injeção $1,0 \mu \mathrm{L}$; íons monitorados $\mathrm{m} / \mathrm{z} 62$ e 75 
Tabela 3. Relação sinal/ruído para diferentes concentrações de carbamato de etila em etanol $40 \%$ e em aguardente de cana por CG-EM

\begin{tabular}{lc}
$\begin{array}{l}\text { Concentração de carbamato de } \\
\text { etila }(\mu \mathrm{g} / \mathrm{L}) \text { em }\end{array}$ & $\begin{array}{c}\text { Relação sinal ruído (média } \pm \\
\text { desvio padrão) }\end{array}$ \\
\hline etanol $40 \%$ & $7,1 \pm 0,9$ \\
30 & $6,5 \pm 1,3$ \\
20 & $3,7 \pm 2,0$ \\
10 & $1,7 \pm 0,5$ \\
5 & \\
aguardente de cana & $7,4 \pm 0,9$ \\
50 & $3,2 \pm 0,1$ \\
30 & $1,8 \pm 0,1$ \\
20 & \\
\hline
\end{tabular}

GC-EM em coluna Carbowax 20M (25 m x 0,22 mm x 0,25 $\mu \mathrm{m})$; temperaturas do injetor e da interface de 250 e $230{ }^{\circ} \mathrm{C}$, respectivamente; temperatura do forno de $90{ }^{\circ} \mathrm{C} / 2 \mathrm{~min}$, elevada a $10{ }^{\circ} \mathrm{C} / \mathrm{min}$ até $150{ }^{\circ} \mathrm{C}(0 \mathrm{~min})$ e elevada a $40^{\circ} \mathrm{C} / \mathrm{min}$ até $230{ }^{\circ} \mathrm{C}(10 \mathrm{~min})$; gás de arraste - He a 1,5 mL/min; volume de injeção $1,0 \mu \mathrm{L}$; íons monitorados $m / z$, 62 e 75 .

Como não foi possível obter uma matriz sem o carbamato de etila, a recuperação foi determinada pelo método de adição, ou seja, incorporando quantidades conhecidas de padrão em amostras de aguardente. Os percentuais de recuperação e os coeficientes de variação (Tabela 2) estão de acordo com o Codex, ${ }^{32}$ para analitos em concentrações de 10 a $100 \mu \mathrm{g} / \mathrm{L}$ (70 a $110 \%$, e $20 \%$, respectivamente) e maiores que $100 \mu \mathrm{g} / \mathrm{L}$ ( 80 a $110 \%$, e $15 \%$, respectivamente). Estes resultados demonstram a exatidão e precisão do método.

\section{Teores de carbamato de etila em aguardentes de cana de Minas Gerais}

Os teores de carbamato de etila nas 71 amostras de aguardente de cana produzidas e/ou engarrafadas em Minas Gerais variaram entre as amostras, sendo que apenas uma apresentou teor abaixo do limite de quantificação do método (Figura 1S). De acordo com a análise estatística descritiva, os teores de carbamato de etila variaram de 33 a $2609 \mu \mathrm{g} / \mathrm{L}$, sendo a média e a mediana iguais a 893 e $787 \mu \mathrm{g} / \mathrm{L}$, respectivamente. A maioria das amostras (35\%) apresentou teores de carbamato de etila entre 500 e $1000 \mu \mathrm{g} / \mathrm{L}$ (Figura 3) e 12\% continham

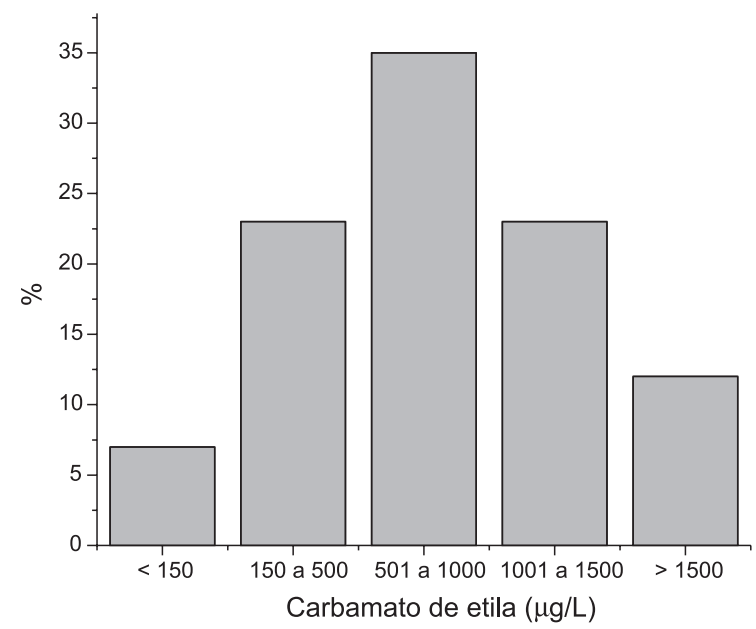

Figura 3. Histograma dos teores de carbamato de etila em amostras de aguardente de cana por CG-EM coletadas no mercado consumidor de Belo Horizonte, MG, no período de maio de 2003 a março de 2004 teores 10 vezes maiores que o estipulado pela legislação

Ao agrupar as amostras em função da região de origem, observouse diferença significativa quanto ao teor de carbamato de etila. Teores significativamente maiores foram encontrados na região Noroeste, os quais não diferiram significativamente dos teores da região Metropolitana. Esta, por sua vez, não diferiu das outras regiões. Todas as regiões apresentaram elevados percentuais $(75 \%)$ de não atendimento ao limite de $150 \mu \mathrm{g} / \mathrm{L}$, sendo que na região Noroeste, o percentual de não atendimento foi de $100 \%$.

São poucos os dados disponíveis na literatura sobre carbamato de etila em aguardente de cana. Boscolo et al. ${ }^{31}$ e Andrade Sobrinho et $a l .{ }^{30}$ indicaram faixas mais amplas de carbamato de etila (42 a 5689 $\mu \mathrm{g} / \mathrm{L}$ em 84 amostras e 13 a $5700 \mu \mathrm{g} / \mathrm{L}$ em 127 amostras, respectivamente) que as encontradas neste estudo, entretanto os teores médios foram similares. Por outro lado, Bruno et al., ${ }^{19}$ Yonamine et al. ${ }^{35} \mathrm{e}$ Lelis $^{36}$ obtiveram teores mais baixos (nd a $714 \mu \mathrm{g} / \mathrm{L}$, nd a $1500 \mu \mathrm{g} / \mathrm{L}$ e 20 a $948 \mu \mathrm{g} / \mathrm{L}$, respectivamente) em produtos recém destilados ou disponíveis no mercado de diferentes cidades do Brasil. Em todos estes estudos, entretanto, um grande percentual de amostras não atenderia ao limite de $150 \mu \mathrm{g} / \mathrm{L}$, ressaltando a importância de se determinarem os mecanismos envolvidos na formação desta substância, para que intervenções possam ser feitas no processamento de modo a minimizar os teores no produto final.

\section{Correlação entre os teores de carbamato de etila e outros parâmetros de qualidade da aguardente de cana}

Estudos de correlação entre os teores de carbamato de etila e o grau alcoólico, $\mathrm{pH}$ e teores de cobre, determinados nas mesmas amostras, ${ }^{5}$ indicaram a não existência de correlação significativa em nível de $5 \%$. Algumas variáveis não controladas neste estudo, como amostras com diferentes condições e tempos de armazenamento, tempo de envelhecimento variado, dentre outros, provavelmente contribuíram para estes resultados.

De modo geral, os teores de carbamato de etila encontrados foram elevados e variaram entre amostras e entre regiões. $\mathrm{O}$ fato de algumas amostras não apresentarem quantidades detectáveis deste composto indica que é possível produzir aguardente sem a presença de quantidades elevadas de carbamato de etila. Entretanto, para tal, estudos devem ser feitos ao longo do processo de produção da aguardente para determinar os pontos críticos de controle e propor alternativas tecnológicas para minimizar a presença desta substância. Desta forma, um produto seguro e de boa qualidade do ponto de vista toxicológico seria produzido, atendendo aos padrões estabelecidos na legislação nacional e internacional.

\section{CONCLUSÕES}

O método para determinação de carbamato de etila por GC-EM foi otimizado e validado, demonstrando especificidade, seletividade, linearidade e limite de quantificação de $30 \mu \mathrm{g} / \mathrm{L}$. Além disto, o método não requer etapas de extração e purificação da amostra.

Os teores de carbamato de etila em amostras de aguardente produzidas em Minas Gerais variaram de <30 a $2609 \mu \mathrm{g} / \mathrm{L}$. Apenas $7 \%$ do total das amostras apresentaram teores inferiores ao limite estipulado pela legislação $(150 \mu \mathrm{g} / \mathrm{L})$. Houve diferença significativa quanto aos teores médios encontrados para as amostras de diferentes regiões do estado. Não foi observada correlação significativa entre os teores de carbamato de etila, $\mathrm{pH}$, cobre e grau alcoólico para as amostras analisadas.

\section{MATERIAL SUPLEMENTAR}

De acordo com a Figura 1S, apenas 7\% das amostras atenderam ao limite de $150 \mu \mathrm{g} / \mathrm{L}$ de carbamato de etila estipulado pela legislação 
vigente. O material suplementar está disponível gratuitamente em http://quimicanova.sbq.org.br, na forma de arquivo PDF.

\section{AGRADECIMENTOS}

Ao CNPq e à Fapemig pelo apoio financeiro e ao Laboratório de Ensaios de Combustíveis - LEQ, UFMG pelo apoio analítico.

\section{REFERÊNCIAS}

1. Cardoso, D. R.; Lima-Neto, B. S.; Franco, D. W.; Nascimento, R. F.; Quim. Nova 2003, 26, 165.

2. http//www.udop.com.br/diversas/materias.htm, acessada em Março 2003.

3. Azevedo, S. M.; Cardoso, M. G.; Pereira, M. E.; Ribeiro, C. F. S.; Silva, V. F.; Aguiar, F. C.; Ciênc. Agrotecnol. 2003, 27, 618.

4. Silva, R. M. C.; Almeida, E.; Valencia, E. P. E.; Nascimento Filho, V. F.; J. Radioanal. Nucl. Chem. 2004, 260, 3.

5. Labanca, R. A.; Glória, M. B. A.; Gouveia, V. J. P.; Afonso, R. J. C. F.; Quim. Nova 2006, 29, 1110.

6. Beland, F. A.; Benson, R. W.; Mellick, P. W.; Kovatch, R. M.; Roberts, M. K.; Fang, J. L.; Doerge, D. R.; Food Chem. Toxicol. 2005, 43, 1.

7. IARC Monographs on the evaluation of carcinogenic risk of chemicals to humans; Geneva, 1987, 7, 43.

8. Hernandez, L. G.; Forkert, P. -G.; Int. J. Cancer 2007, 120, 1426.

9. Park, S. -K.; Kim, C. T.; Lee, J. -W.; Jhee, O. H.; Om, A. S.; Kang, J. S.; Moon, T. W.; Food Control 2007, 18, 975.

10. Lawrence, J. F.; Page, B. D.; Conacher, H. B. S.; Adv. Environ. Sci. Technol. 1990, 23, 457.

11. FDA. US Food and Drug Administration, Center for Food Safety and Nutrition, 2000. Available from www.cfsan.fda.gov/ frf/ecintro.html.

12. Brasil. Instrução normativa no. 13, de 29 de junho de 2005, Diário Oficial da União, 30/06/2005, s.1, p. 3, Brasília.

13. Ordunã, R. M.; Liu, S. Q.; Patchett, M. L.; Pilone, G. J.; FEMS Microbiol. Lett. 2000, 183, 31.

14. Nagato, L. A. A. F.; Novaes, F. V.; Penteado, M. V. C.; Bol. SBCTA 2003, 37,40 .

15. Arena, M. E.; Nadra, M. C. M.; Res. Microbiol. 2005, 156, 858.
16. Uthurry, C. A.; Lepe, J. A. S.; Lombardero, J.; Hierro, J. R. G.; Food Chem. 2006, 94, 262.

17. Aresta, M.; Boscolo, M.; Franco, D. W.; J. Agric. Food Chem. 2001, 49 , 1819.

18. Mackenzie, W. M.; Clyne, H.; Macdonald, L. S.; J. Inst. Brew. 1990, 96 , 223.

19. Bruno, S. N. F.; Vaistman, D. S.; Kunigami, C. N.; Brasil, M. G.; Food Chem. 2007, 104, 1345.

20. Eurachem; The fitness for purpose of analytical methods. A laboratory guide to method validation and related topics, 1988.

21. Ough, C. S.; J. Agric. Food Chem. 1976, 24, 328.

22. Brumley, W. C.; Canas, B. J.; Perfetti, G. A.; Mossoba, M. M.; Sphon, J. A.; Corneliussen, P. E.; Anal. Chem. 1988, 60, 975.

23. Clegg, B. S.; Frank, R.; J. Agric. Food Chem. 1988, 36, 502.

24. Aylott, R. I.; Mcneish, A. S.; Walker, D. A.; J. Inst. Brew. 1987, 93, 382.

25.Aylott, R.; Cochrane, C.; Leonard, M. J.; Macdonald, L. S.; Mackenzie, W. M.; Mcneish, A. S.; Walker, D. A.; J. Inst. Brew. 1990, 96, 213.

26. Dennis, M. J.; Howarth, N.; Key, P. E.; Pointer, M.; Massey, R. C.; Food Addit. Contam. 1989, 6, 383.

27. Mossoba, M. M.; Chen, J. T.; Brumley, W. C.; Page, S. W.; Anal. Chem. 1988, 60, 945.

28. Dyer, R. H.; J. AOAC Int. 1994, 77, 64.

29. Matsudo, T.; Aoki, T.; Abe, K.; Fukuta, N.; Higuchi, T.; Sasaki, M.; Uchida, K.; J. Agric. Food Chem. 1993, 41, 352.

30. Andrade-Sobrinho, A. L.; Boscolo, M.; Lima-Neto, B. S.; Franco, D. W.; Quim. Nova 2002, 25, 1074.

31. Boscolo, M.; Tese de Doutorado, Universidade de São Carlos, Brasil, 2002.

32. Codex Alimentarius; Resíduos de medicamentos veterinários en los alimentos, $2^{\text {a }}$ ed., Roma, 1993, vol. 3.

33. http://www.ibge.gov.br, acessada em Março 2006.

34. Association of Official Analytical Chemists; Official Methods of Analysis, Arlington: AOAC, 1995

35. Yonamine, M.; Nagato, L. A. F.; Penteado, M. V. C.; Silva, O. A.; Alimentaria 2000, 311, 37.

36. Lelis, V. G.; Dissertação de Mestrado, Universidade Federal de Viçosa, Brasil, 2006. 


\section{DETERMINAÇÃO DE CARBAMATO DE ETILA EM AGUARDENTES DE CANA POR CG-EM}

Renata Adriana Labanca e Maria Beatriz Abreu Glória*

Departamento de Alimentos, Faculdade de Farmácia, Universidade Federal de Minas Gerais, Av. Antônio Carlos, 6627, $31270-901$ Belo Horizonte - MG, Brasil

\section{Robson José de Cássia Franco Afonso}

Departamento de Química, Universidade Federal de Ouro Preto, R. Diogo de Vasconcelos, 122, 35400-000 Ouro Preto - MG, Brasil

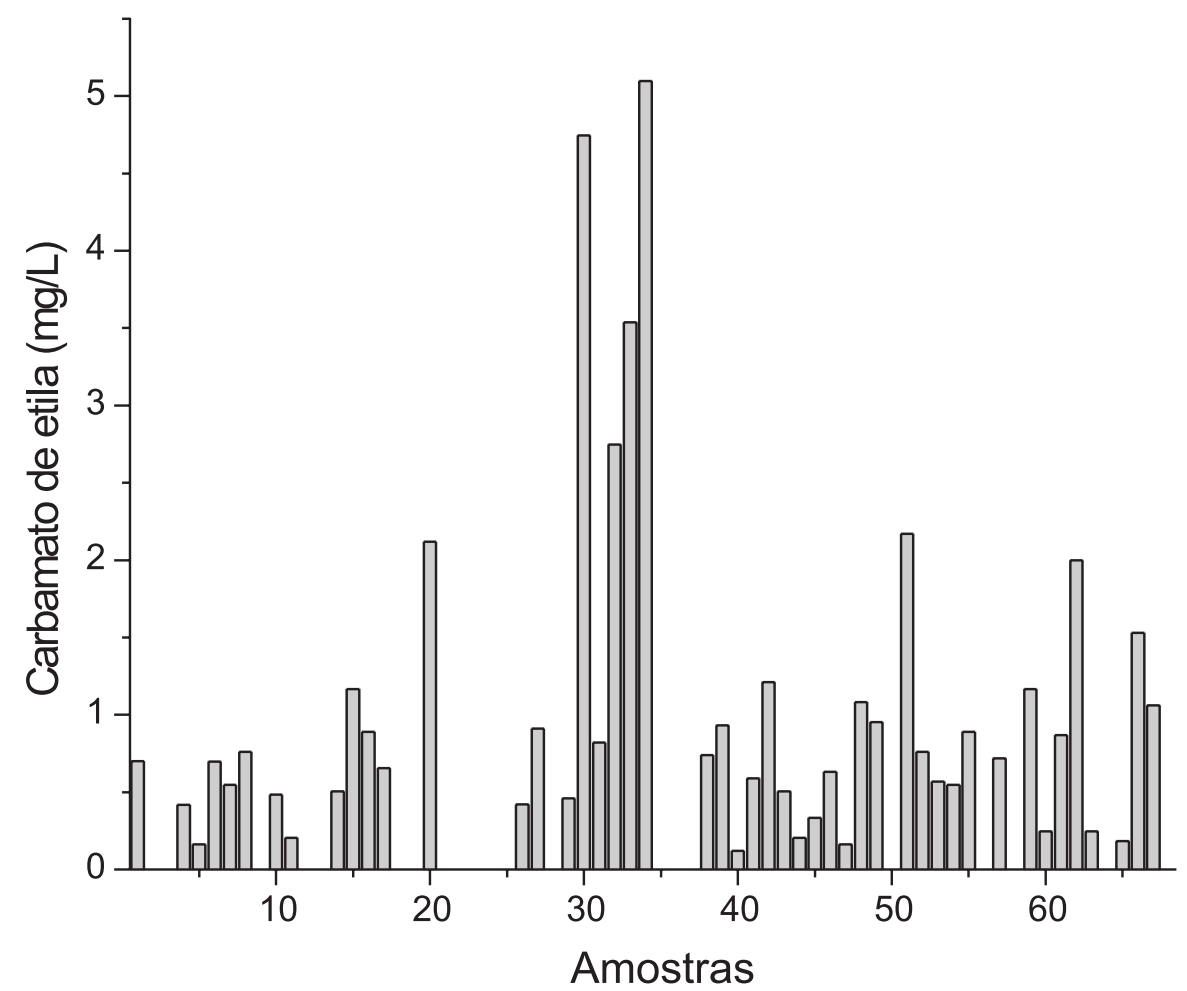

Figura 1S. Teores de carbamato de etila em 71 amostras de aguardente de cana coletadas em Belo Horizonte, MG, de maio de 2003 a março de 2004 e agrupadas em função da região de origem 